\title{
Forme Fruste Keratoconus Imaging and Validation via Novel Multi-Spot Reflection Topography
}

\author{
Anastasios John Kanellopoulos ${ }^{a, b} \quad$ George Asimellis ${ }^{a}$ \\ ${ }^{a}$ LaserVision.gr Eye Institute, Athens, Greece; ${ }^{b}$ New York University Medical School, \\ New York, N.Y., USA
}

\section{Key Words}

Light-emitting diode Cassini - Light-emitting diode topography · Diagnosis of keratoconus . Forme fruste keratoconus · Point-source topography · Pentacam HR - Placido topography . Surface Asymmetry Index · Surface Regularity Index · Index of Surface Variance · Index of Height Decentration · Differential topography · Color-point topography

\begin{abstract}
Background/Aims: This case report aims to evaluate safety, efficacy and applicability of anterior surface imaging in a patient with forme fruste keratoconus (FFKC) based on a novel multi-spot, multicolor light-emitting-diode (LED) tear film-reflection imaging technology Case Description: A 45-year-old male patient, clinically diagnosed with FFKC, with highly asymmetric manifestation between his eyes, was subjected to the multicolor-spot reflection topography. We investigated elevation and sagittal curvature maps comparatively with the multicolor-spot reflection topographer, a Placido topographer and a Scheimpflug imaging system. For the right eye, steep and flat keratometry values were 41.92 and $41.05 \mathrm{D}$ with the multicolor spot-reflection topographer, 42.30 and $42.08 \mathrm{D}$ with the Placido, and 41.95 and $41.19 \mathrm{D}$ with the Scheimpflug system. For the left eye, steep and flat keratometry values were 41.86 and $41.19 \mathrm{D}$ with the multicolor spot-reflection topographer, 42.06 and $41.66 \mathrm{D}$ with the Placido topographer, and 41.96 and $41.66 \mathrm{D}$ with the Scheimpflug camera. Average repeatability of the keratometry measurements was $\pm 0.35 \mathrm{D}$ for the multicolor spot-reflection topographer, $\pm 0.30 \mathrm{D}$ for the Placido, and $\pm 0.25 \mathrm{D}$ for the Scheimpflug camera. Very good agreement between the instruments was demonstrated on the elevation and curvature maps. Conclusion: The ease of use and the comparable results offered by the multicolor spotreflection topographer, in comparison to established Placido and Scheimpflug imaging, as well as the increased predictability that may be offered by the multicolor spot-reflection topographer, may hold promise for wider clinical application, such as screening of young
\end{abstract}

A. John Kanellopoulos, MD

LaserVision.gr Eye Institute, 17 Tsocha Street

GR-11521 Athens (Greece)

E-Mail ajk@brilliantvision.com 
Kanellopoulos et al.: Forme Fruste Keratoconus Imaging and Validation via Novel Multi-Spot Reflection Topography

adults for early keratoconus and, in a much wider perspective, potential candidates for laser corneal refractive surgery.

(c) 2013 S. Karger AG, Basel

\section{Introduction}

Topography systems with color-coded point-source reflection forward ray tracing have been proposed [1] as alternatives to Placido ring projection [2], enabling, among other, proper identification of radial, rather than only contour topographic changes [3]. The VU topographer (Vrije Universiteit Medical Center, Amsterdam, The Netherlands) [1] introduced a different approach, with color-coded squares in a chess-pattern array projected on the cornea instead of the Placido rings [4]. The algorithm for the surface reconstruction employed data from the pattern crossing points to eliminate source-image mismatch, enabling one-to-one match. In principle, this color-coded topographer was found to be efficient in reconstructing the non-rotationally symmetric anterior corneal surface [5].

The Cassini (i-Optics, The Hague, The Netherlands) topography system is a novel topographer employing multi-spot (up to 700), multi-color (red, yellow and green) LED tear filmreflection-imaging, following the steps of the VU topographer. The difference is that instead of a limited number of color-coded squares, there are hundreds of LED spots imaged on the cornea. Image processing algorithm locates feature points in the reflection image and accounts for smearing and deformation in irregular corneas. The system has been recently introduced [6] and has received US Food and Drug Administration (FDA) approval for clinical use in corneal topography.

Due to the novelty of this system, its clinical validation and implications have yet to be investigated. To the best of our knowledge, there is no other publication in the peerreviewed literature investigating the clinical applicability of this new device. The scope of this case report is to examine the clinical feasibility of this newly introduced point-source color-coded LED array corneal topographer in a case of forme fruste keratoconus (FFKC). FFKC has been described by Amsler in 1961 [7] as 'an incomplete, abortive or unusual form of a syndrome or disease'.

\section{Case Report}

We present the case of a 45-year-old male subject diagnosed with topographic and topometric asymmetrically manifested FFKC $[8,9]$ between his two eyes. The patient, a resident of Sweden, father of a 17-year-old adolescent who had developed keratoconus, attended treatment with the Athens Protocol procedure of his son in our institution [10]. Due to the known hereditary nature of the disease [11], our keratoconus protocol involves examination of next-of-kin whenever possible.

Three imaging modalities were studied in this work. Scheimpflug imaging was provided by the WaveLight Oculyzer II (Alcon Surgical, Fort Worth, Tex., USA), a Pentacam HR (highresolution) camera providing corneal pachymetry and tomography imaging (covering a 9$\mathrm{mm}$ diameter area). The system, running on Examination Software version 1.17r47, additionally provides keratometry, topographic keratoconus classification and computes irregularity keratoconus indices, namely the Index of Surface Variance (ISV) and the Index of Height Decentration (IHD) $[12,13]$. Placido imaging was provided by the WaveLight Allegro Topolyzer (Alcon Surgical). The Topolyzer is a wide cone Placido system covering an approximately $8.5 \mathrm{~mm}$-diameter area with 22 concentric rings for the detection of up to 
Kanellopoulos et al.: Forme Fruste Keratoconus Imaging and Validation via Novel Multi-Spot Reflection Topography

22,000 elevation points. Both systems are incorporated in our keratoconus screening protocol and are employed in the topography-guided treatment in conjunction with accelerated cross-linking [14].

The Cassini (running on software version 1.1.1) was employed to provide anterior surface imaging. The system produces anterior elevation, tangential and axial curvature, and refractive power three-dimensional maps covering approximately a 7.5-mm diameter area. The report provides keratometry data (steep and flat K, simulated astigmatism), and computes topographic and keratoconus indices, namely the Surface Asymmetry Index (SAI) and the Surface Regularity Index (SRI) [15]. In addition, an aberrations report is provided, according to the Zernike nomenclature. Four consecutive scans in each case were obtained by all systems.

The right eye (OD), the more symptomatic to the patient, had uncorrected distance visual acuity of $20 / 25$ and manifest refraction of plano $+0.50 \times 45^{\circ}$. Corrected distance visual acuity with this prescription was $20 / 20$. In addition to the complete ocular examination, the FFKC diagnosis was further confirmed by Scheimpflug topometry and Placido topography. Data supporting this diagnosis include thinnest local corneal thickness of $492 \mu \mathrm{m}$, and an asymmetric posterior surface elevation, indicating a minimum depression of $-31 \mu \mathrm{m}$ located superiorly and a maximum protrusion of $+25 \mu \mathrm{m}$ located inferiorly. However, this eye (as well as the less affected OS) was classified as nonkeratoconic according to the Scheimpflug imaging-provided topographic keratoconus classification. [13]

The left eye (OS), the less affected, had uncorrected distance visual acuity of 20/20 and manifest refraction of plano. The rather symmetric posterior elevation, as obtained by the Pentacam, indicated a minimum superior depression of $-25 \mu \mathrm{m}$, minimum inferior depression of $-17 \mu \mathrm{m}$, and a maximum local inferior protrusion (within the center 2-mm area) of $+13 \mu \mathrm{m}$. The thinnest local corneal thickness was $496 \mu \mathrm{m}$.

Repeatability results for flat and steep keratometry, astigmatism and meridian axis as measured by all three modalities, were assessed by the standard deviation of data from the four consecutive acquisitions. Descriptive statistics for the above parameters are reported in table 1. Results from the Cassini keratoconus indices (SAI and SRI) and Scheimplug-derived keratoconus indices (ISV and IHD) [13] are reported in table 2. Results from anterior elevation data as measured by the Cassini and the Pentacam are reported in table 3.

Comparative examples of anterior elevation maps from both modalities employed in the study for the more affected OD and the less affected OS eye are provided in fig. 1, b, respectively. In addition, comparative examples of anterior surface curvature maps for the evidently asymmetric OD and the less affected OS are provided in fig. $2 \mathrm{a}, \mathrm{b}$, respectively. Fig. 3 presents the results of the Placido topography system imaging for the right (top) and left (bottom) eye.

This study adhered to the tenets of the Declaration of Helsinki and was approved by the Ethics Committee of our Institution. Informed consent was obtained from the subject at the time of the first clinical visit.

\section{Discussion}

An important practical task for clinicians is to improve the sensitivity of their screening methods for identifying patients with mild manifestations of keratoconus and prevent iatrogenic keratectasia. The term FFKC has been proposed [16] for cases that may exhibit subtle topographic characteristics suggestive of an early subclinical keratoconus but that are 
Kanellopoulos et al.: Forme Fruste Keratoconus Imaging and Validation via Novel Multi-Spot Reflection Topography

not pronounced enough to reach the threshold of suspicion with automated classification systems [17].

Therefore, this case presents with inherent challenges to be met by the diagnostic devices. In this work, we examined the clinical feasibility of this newly introduced point-source color-coded LED array corneal topography in a case of FFKC in comparison to the established methods of clinical evaluation, namely Placido topography and Scheimpflug topometry.

All three devices were successful in producing accurate corneal imaging, with correlated information. There is very good agreement in both eyes by all instruments regarding keratometry and astigmatism, as well as anterior elevation data. Repeatability was at similar levels for all devices on every pertinent parameter measured (tables 1-3). There is also very good similarity and agreement between the surface elevation maps (fig. 1) and the curvature maps (fig. 2, 3), in which the more apparent (OD) and the less-evident (OS) refractive asymmetries of the anterior surface along the superior-inferior line are clearly recognized by all three clinical diagnostic devices.

\section{Conclusion}

The ease of use and the comparable results offered by the LED topographer, in comparison to established Scheimpflug imaging and Placido topography systems, as well as the increased predictability that may be offered by the LED corneal imaging, may hold promise for wider clinical application, such as screening of young adults for early keratoconus and, in a much wider perspective, potential candidates for laser corneal refractive surgery.

\section{Disclosure Statement}

A.J.K. holds consultant/advisory positions with Alcon/WaveLight, Avedro, and Oculus, and G.A. with Alcon/WaveLight.

\section{References}

-1 Vos FM, van der Heijde RGL, Spoelder HJW, van Stokkum IHM, Groen FCA: A new instrument to measure the shape of the cornea based on pseudorandom color coding. IEEE Trans Instrum Meas 1997;46:794-7-797.

-2 Snellenburg JJ, Braaf B, Hermans EA, van der Heijde RG, Sicam VA: Forward ray tracing for image projection prediction and surface reconstruction in the evaluation of corneal topography systems. Opt Express 2010;18:19324-19338.

-3 Rand RH, Howland HC, Applegate RA: Mathematical model of a Placido disk keratometer and its implications for recovery of corneal topography. Optom Vis Sci 1997;74:926-930.

-4 Sicam VA, Snellenburg JJ, van der Heijde RG, van Stokkum IH: Pseudo forward ray-tracing: a new method for surface validation in cornea topography. Optom Vis Sci 2007;84:915-923.

-5 Sicam VA, van der Heijde RG: Topographer reconstruction of the nonrotation-symmetric anterior corneal surface features. Optom Vis Sci 2006;83:910-918.

6 Weikert MP, Koch DD, Wang D: Evaluation of Corneal Topography Based on Color LED Technology. ASCRS Symposium \& Congress 2013, San Francisco, USA.

$\checkmark 7$ Amsler M: The 'forme fruste' of keratoconus (in German). Wien Klin Wochenschr 1961;73:842-843.

8 Saad A, Gatinel D: Topographic and tomographic properties of forme fruste keratoconus corneas. Invest Ophthalmol Vis Sci 2010;51:5546-5555.

-9 Smadja D, Touboul D, Cohen A, Doveh E, Santhiago MR, Mello GR, Krueger RR, Colin J: Detection of subclinical keratoconus using an automated decision tree classification. Am J Ophthalmol 2013;156:237246.e1 
Kanellopoulos et al.: Forme Fruste Keratoconus Imaging and Validation via Novel Multi-Spot Reflection Topography

10 Kanellopoulos AJ, Asimellis G: Keratoconus management: long term stability of topography-guided normalization combined with high fluence CXL stabilization (The Athens Protocol). J Refract Surg 2013, accepted for publication.

-11 Wheeler J, Hauser MA, Afshari NA, Allingham RR, Liu Y: The genetics of keratoconus: a review. Reprod Syst Sex Disord 2012;(suppl 6). doi:pii: 001.

12 Correia FF, Ramos I, Lopes B, Salomão MQ, Luz A, Correa RO, Belin MW, Ambrósio R: Topometric and tomographic indices for the diagnosis of keratoconus. Int J Kerat Ectatic Dis 2012;1:92-99.

-13 Kanellopoulos AJ, Asimellis G: Revisiting keratoconus diagnosis and progression classification based on evaluation of corneal asymmetry indices, derived from Scheimpflug imaging in keratoconic and suspect cases. Clin Ophthalmol 2013;7:1339-1348.

14 Kanellopoulos AJ, Asimellis G: Comparison of Placido disc and Scheimpflug image-derived topographyguided excimer laser surface normalization combined with higher fluence CXL: the Athens Protocol, in progressive keratoconus. Clin Ophthalmol 2013;7:1385-1396.

-15 Smolek MK, Klyce SD: Current keratoconus detection methods compared with a neural network approach. Invest Ophthalmol Vis Sci 1997;38:2290-2299.

Klyce SD: Chasing the suspect: keratoconus. Br J Ophthalmol 2009;93:845-847.

Gatinel D, Saad A: The challenges of the detection of subclinical keratoconus at its earliest stage. Int J Kerat Ectatic Dis 2012;1:36-43. 


\begin{tabular}{l|l}
\hline DOI: $10.1159 / 000356123$ & $\begin{array}{l}\text { C } 2013 \text { S. Karger AG, Basel } \\
\text { www.karger.com/cop }\end{array}$ \\
\hline
\end{tabular}

Kanellopoulos et al.: Forme Fruste Keratoconus Imaging and Validation via Novel Multi-Spot Reflection Topography

Table 1. Descriptive statistics for flat and steep keratometry, astigmatism, meridian axis $\left({ }^{\circ}\right)$ and maximum as measured by the Cassini, the Scheimpflug Pentacam and the Topolyzer Placido devices

\begin{tabular}{|c|c|c|c|c|c|c|c|}
\hline & $\begin{array}{l}\text { Steep K, } \\
\mathrm{mm}\end{array}$ & $\begin{array}{l}\text { Steep K, } \\
\text { D }\end{array}$ & $\begin{array}{l}\text { Axis } \\
\circ\end{array}$ & $\begin{array}{l}\text { Flat K, } \\
\mathrm{mm}\end{array}$ & $\begin{array}{l}\text { Flat K, } \\
\text { D }\end{array}$ & $\begin{array}{l}\text { Axis } \\
\circ\end{array}$ & $\begin{array}{l}\text { Astigmatism, } \\
\text { D }\end{array}$ \\
\hline \multicolumn{8}{|l|}{ Cassini } \\
\hline \multicolumn{8}{|l|}{ OD } \\
\hline Average & 8.06 & 41.92 & 184 & 8.23 & 41.05 & 94 & -0.87 \\
\hline StDev & 0.04 & 0.21 & 9 & 0.03 & 0.16 & 9 & 0.09 \\
\hline Min & 8.07 & 41.46 & 175 & 8.15 & 41.05 & 85 & -0.96 \\
\hline Max & 8.15 & 41.87 & 195 & 8.23 & 41.46 & 105 & -0.46 \\
\hline \multicolumn{8}{|l|}{ OS } \\
\hline Average & 8.07 & 41.86 & 135 & 8.12 & 41.62 & 45 & -0.23 \\
\hline StDev & 0.04 & 0.19 & 11 & 0.02 & 0.11 & 11 & 0.13 \\
\hline Min & 8.04 & 41.61 & 123 & 8.09 & 41.51 & 33 & -0.41 \\
\hline Max & 8.12 & 42.02 & 148 & 8.14 & 41.76 & 58 & -0.10 \\
\hline \multicolumn{8}{|l|}{ Pentacam } \\
\hline \multicolumn{8}{|l|}{ OD } \\
\hline Average & 8.06 & 41.95 & 156.25 & 8.20 & 41.19 & 66.25 & -0.75 \\
\hline StDev & 0.07 & 0.37 & 9.71 & 0.08 & 0.42 & 9.71 & 0.55 \\
\hline Min & 7.99 & 41.46 & 143 & 8.10 & 40.71 & 53 & -1.47 \\
\hline Max & 8.15 & 42.29 & 164 & 8.30 & 41.71 & 74 & -0.15 \\
\hline \multicolumn{8}{|l|}{ OS } \\
\hline Average & 8.05 & 41.96 & 149.50 & 8.11 & 41.66 & 59.50 & -0.30 \\
\hline StDev & 0.01 & 0.05 & 11.68 & 0.02 & 0.09 & 11.68 & 0.12 \\
\hline Min & 8.04 & 41.92 & 135 & 8.09 & 41.56 & 45 & -0.47 \\
\hline Max & 8.06 & 42.02 & 162 & 8.13 & 41.76 & 72 & -0.21 \\
\hline \multicolumn{8}{|l|}{ Topolyzer } \\
\hline \multicolumn{8}{|l|}{ OD } \\
\hline Average & 7.99 & 42.30 & 144.50 & 8.03 & 42.08 & 54.50 & -0.22 \\
\hline StDev & 0.02 & 0.09 & 3.87 & 0.04 & 0.23 & 3.87 & 0.28 \\
\hline Min & 7.97 & 42.18 & 141 & 7.99 & 41.76 & 51 & -0.58 \\
\hline Max & 8.01 & 42.39 & 150 & 8.09 & 42.29 & 60 & 0.00 \\
\hline \multicolumn{8}{|l|}{ OS } \\
\hline Average & 8.03 & 42.06 & 149.50 & 8.11 & 41.66 & 59.50 & -0.40 \\
\hline StDev & 0.03 & 0.14 & 11.68 & 0.02 & 0.11 & 11.68 & 0.23 \\
\hline Min & 8.00 & 41.92 & 135 & 8.09 & 41.51 & 45 & -0.62 \\
\hline Max & 8.06 & 42.23 & 162 & 8.14 & 41.76 & 72 & -0.21 \\
\hline
\end{tabular}


Kanellopoulos et al.: Forme Fruste Keratoconus Imaging and Validation via Novel Multi-Spot Reflection Topography

Table 2. Descriptive statistics for the Cassini keratoconus indices (SAI and SRI) and the Pentacam keratoconus indices (ISV and IHD)

\begin{tabular}{lrr}
\hline & SAI & SRI \\
\hline Cassini & & \\
OD & & \\
$\quad$ Average & 0.31 & 0.49 \\
$\quad$ StDev & 0.04 & 0.13 \\
$\quad$ Min & 0.22 & 0.29 \\
$\quad$ Max & 0.31 & 0.58 \\
OS & & \\
$\quad$ Average & 0.27 & -0.02 \\
$\quad$ StDev & 0.04 & 0.09 \\
$\quad$ Min & 0.22 & -0.14 \\
$\quad$ Max & 0.31 & -0.08 \\
\hline$\quad$ ISV & IHD \\
& & \\
Pentacam & & \\
OD Average & 16.75 & 0.012 \\
$\quad$ StDev & 1.71 & 0.001 \\
$\quad$ Min & 15.00 & 0.011 \\
$\quad$ Max & 19.00 & 0.013 \\
OS Average & 11.50 & 0.003 \\
$\quad$ StDev & 1.29 & 0.001 \\
$\quad$ Min & 10.00 & 0.002 \\
\hline$\quad$ & 13.00 & 0.004 \\
\hline
\end{tabular}


Kanellopoulos et al.: Forme Fruste Keratoconus Imaging and Validation via Novel Multi-Spot Reflection Topography

Table 3. Descriptive statistics for corneal elevation data, as obtained by both the Cassini and the Pentacam systems

\begin{tabular}{lrr}
$\begin{array}{l}\text { Cassini } \\
\text { OD }\end{array}$ & \\
$\quad$ Average & 10.50 & -10.50 \\
$\quad$ StDev & 1.29 & 1.73 \\
$\quad$ Min & 9.00 & -13.00 \\
$\quad$ Max & 12.00 & -9.00 \\
OS & & \\
$\quad$ Average & 7.50 & -4.75 \\
$\quad$ StDev & 0.58 & 0.96 \\
$\quad$ Min & 7 & -6 \\
$\quad$ Max & 8 & -4 \\
\hline Pentacam & & \\
OD & & \\
$\quad$ Average & 9.50 & -11.50 \\
StDev & 0.58 & 1.29 \\
$\quad$ Min & 9.00 & -13.00 \\
$\quad$ Max & 10.00 & -10.00 \\
OS & & \\
$\quad$ Average & 7.25 & -5.75 \\
StDev & 0.50 & 1.71 \\
$\quad$ Min & 7.00 & -8.00 \\
$\quad$ Max & 8.00 & -4.00
\end{tabular}

All units are expressed in micrometer. 
Case Reports in

Ophthalmology
Case Rep Ophthalmol 2013;4:199-209

DOI: $10.1159 / 000356123$

Kanellopoulos et al.: Forme Fruste Keratoconus Imaging and Validation via Novel Multi-Spot Reflection Topography
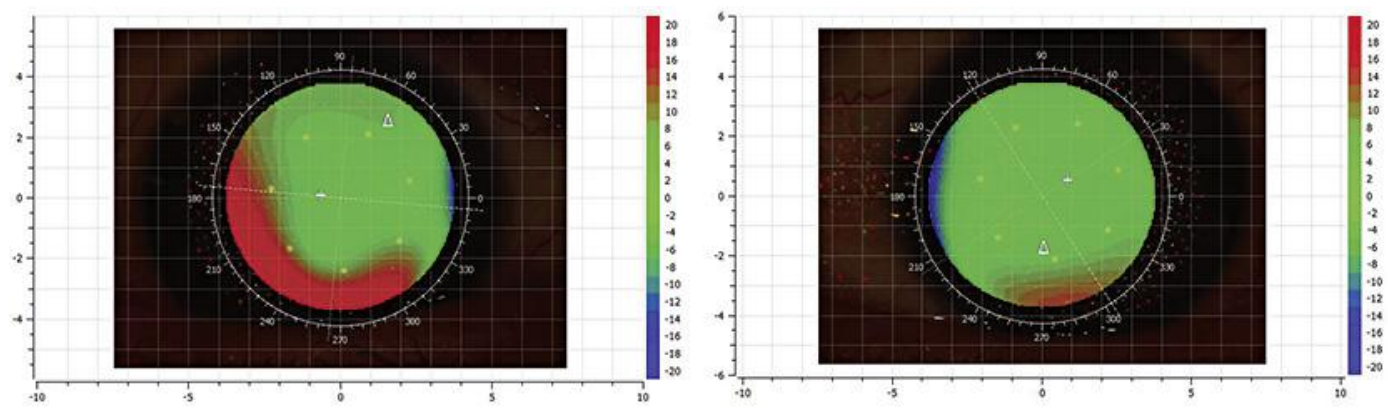

a
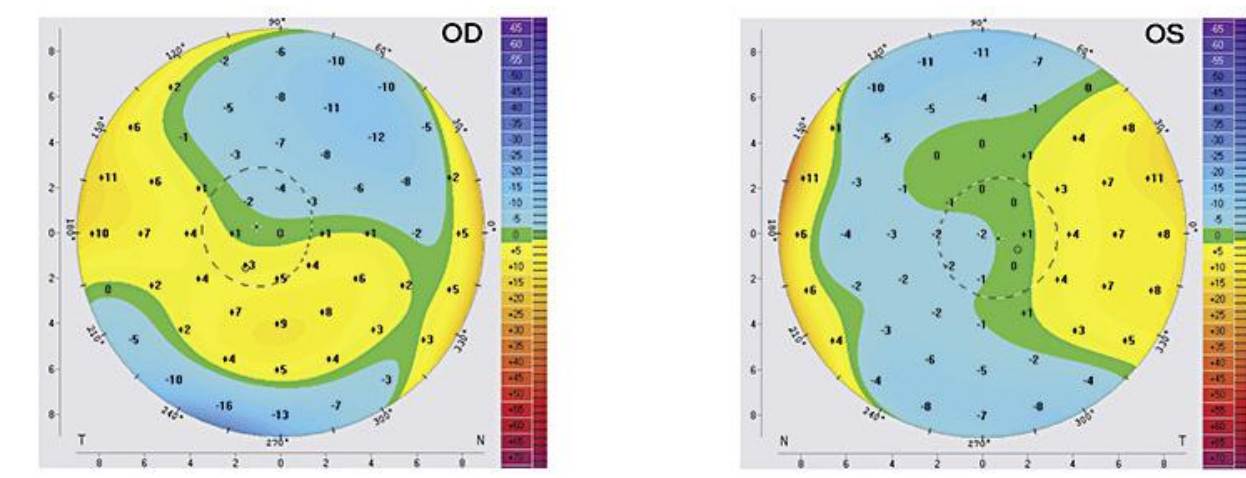

b

Fig. 1. a Top panel: anterior surface elevation map of the OD obtained by the Cassini. Bottom panel: anterior surface elevation map of the OD obtained by the Pentacam. b Top panel: anterior surface elevation map of the OS obtained by the Cassini. Bottom panel: anterior surface elevation map of the OS obtained by the Pentacam. 
Case Reports in

Ophthalmology
Case Rep Ophthalmol 2013;4:199-209

DOI: $10.1159 / 000356123$

Kanellopoulos et al.: Forme Fruste Keratoconus Imaging and Validation via Novel Multi-Spot Reflection Topography
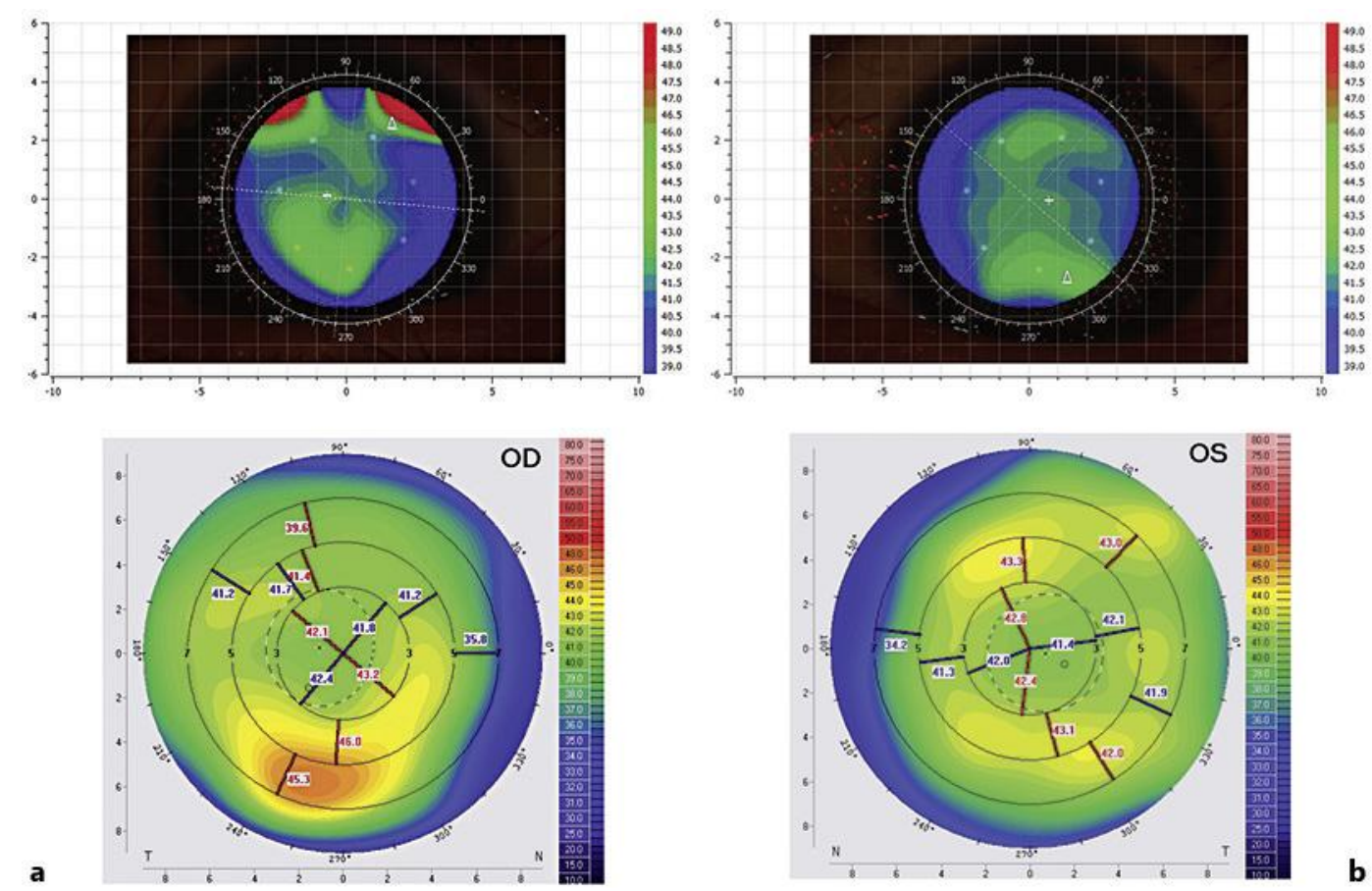

b

Fig. 2. a Top panel: anterior surface tangential curvature map of the OD obtained by the Cassini. Bottom panel: anterior surface tangential curvature map of the OD obtained by the Pentacam. $\mathbf{b}$ Top panel: anterior surface tangential curvature map of the OS obtained by the Cassini. Bottom panel: anterior surface tangential curvature map of the OS obtained by the Pentacam. 


\section{Case Reports in \\ Ophthalmology}

\begin{tabular}{l|l}
\hline \multicolumn{2}{l}{ Case Rep Ophthalmol 2013;4:199-209 } \\
\hline DOI: $10.1159 / 000356123$ & $\begin{array}{l}\text { @ 2013 S. Karger AG, Basel } \\
\text { www.karger.com/cop }\end{array}$ \\
\hline
\end{tabular}

Kanellopoulos et al.: Forme Fruste Keratoconus Imaging and Validation via Novel Multi-Spot Reflection Topography

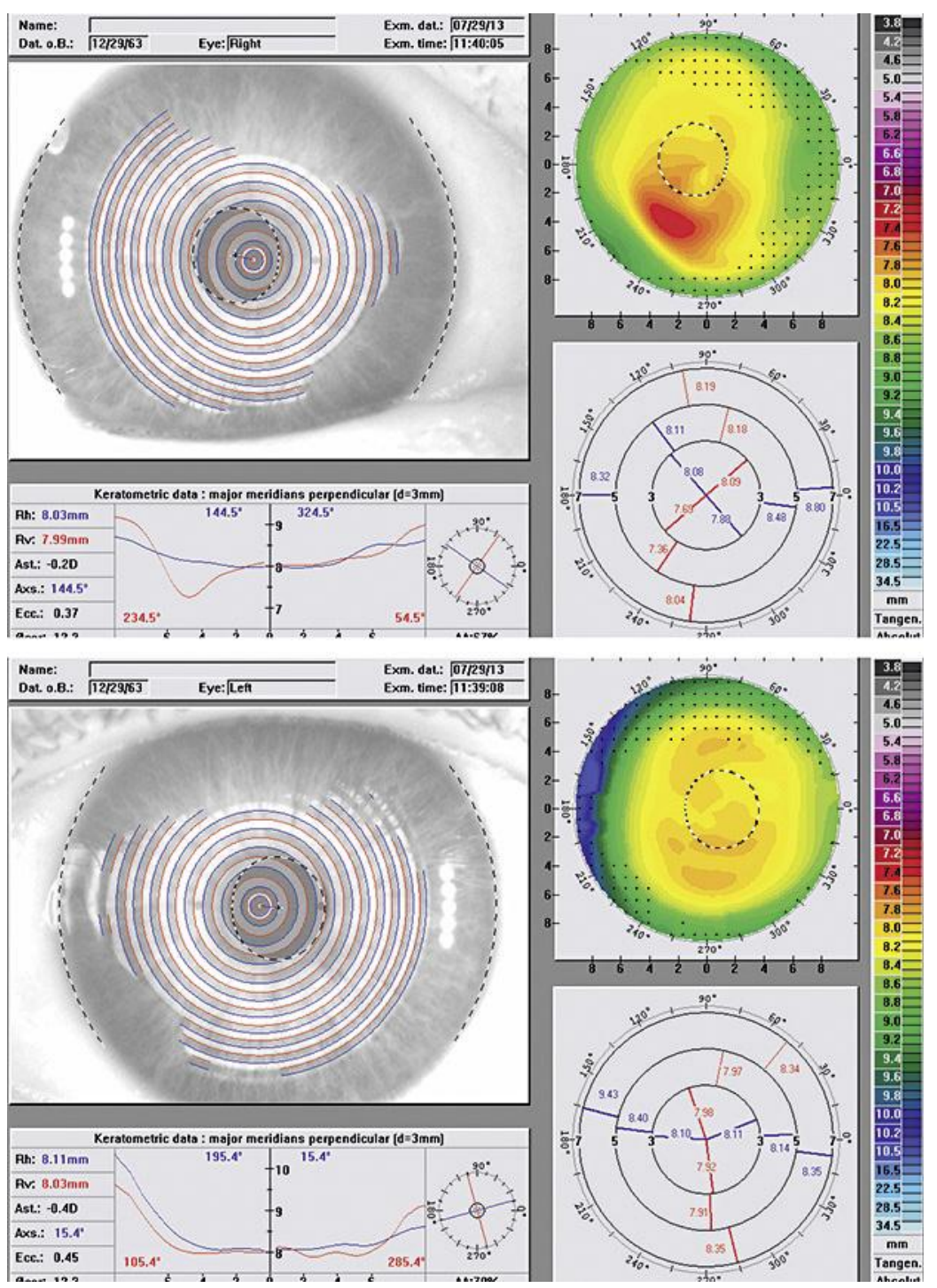

Fig. 3. Placido topography imaging of the OD (top) and OS (bottom). 